\title{
Releitura de pão de queijo: Versão vegetariana com farinha de oliveira
}

\section{Cheese bread rereading: Vegetarian version with olive flour}

\author{
Carolina Dias dos Santos ${ }^{\left(\mathbb{D}^{1}\right.}{ }^{\text {; }}$ Rafaella Câmara Rocha Menezes ${ }^{\left(D^{2}\right.}{ }^{2}$; Simone Morelo Dal Bosco ${ }^{3}{ }^{3}$
}

${ }^{1}$ Graduada em Nutrição; Universidade Federal de Ciências da Saúde de Porto Alegre, Porto Alegre, carolinadiassc@ gmail.com; ${ }^{2}$ Mestre em Ciências da Nutrição; Universidade Federal do Rio Grande do Sul, Porto Alegre. rafaella.crm21@gmail.com ${ }^{3}$ Doutora em Medicina e Ciências da Saúde; Universidade Federal de Ciências da Saúde de Porto Alegre, Programa de Pós Graduação Ciências da Nutrição; Porto Alegre, simonedb @ufcspa.edu.br.

\section{A R T I G O}

Recebido: 23/08/2020

Aprovado: 24/03/2021

Palavras-chave:

Uso de resíduos sólidos

Olea Europaea L.

Vegetarianos

\begin{abstract}
R E S U M O
A oliveira (Olea europaea L.) é composta por folhas que podem ser consumidas, mas costumam ser descartadas pela colheita mecanizada. Uma alternativa para viabilizar o descarte das folhas de oliveiras, é elaborar a farinha de folha de oliveira (FFO) realizando o aproveitamento. Com isso, o objetivo desta pesquisa foi elaborar um pão de queijo vegetariano adicionado de farinha da folha de oliveira a fim de viabilizar uma nova alternativa de aproveitamento tecnológico desse resíduo. Foram desenvolvidas cinco formulações de pães de queijo, alterando-se a quantidade de farinha das folhas de oliveira adicionada $(0,95-2,85 \%)$ e submetidas a avaliação das características sensoriais pelo método de Análise Descritiva Quantitativa. Os resultados demonstraram que, dentre os quinze atributos avaliados, os sabores herbáceo, amargo e de queijo juntamente com o aroma herbáceo apresentaram diferença significativa. Entre os atributos analisados, a FFO contribuiu para o maior sabor amargo dos pães de queijo, seguido de sabor e aroma herbáceo. Conclui-se que foi possível elaborar um pão de queijo vegetariano adicionado de farinha de folha de oliveira como uma alternativa de aproveitamento tecnológico desse resíduo.
\end{abstract}

\section{A B S T R A C T}

Key words:

Solid waste use

Olea Europaea L.

Vegetarians

\begin{abstract}
The olive tree (Olea europaea L.) is made up of leaves that can be consumed, but are usually discarded by mechanized harvesting. An alternative to enable the disposal of olive leaves is to prepare the olive leaf flour (FFO), making use of it. Thus, the objective of this research was to prepare a vegetarian cheese bread added with olive leaf flour in order to make a new alternative for the technological use of this residue feasible. Five cheese bread formulations were developed, changing the amount of flour from the olive leaves added $(0.95-2.85 \%)$ and subjected to the evaluation of sensory characteristics by the method of Quantitative Descriptive Analysis. The results showed that, among the fifteen attributes evaluated, the herbaceous, bitter and cheese flavors together with the herbaceous aroma showed a significant difference. Among the attributes analyzed, the FFO contributed to the greater bitter taste of the cheese breads, followed by the herbaceous flavor and aroma. It was concluded that it was possible to prepare a vegetarian cheese bread added with olive leaf flour as an alternative for the technological use of this residue.
\end{abstract}

\section{INTRODUÇÃO}

Os consumidores estão atentos às características dos alimentos priorizando produtos que além de suprir suas necessidades nutricionais, colaborem para melhor qualidade de vida e bem-estar (BARBOSA; CAZAL, 2018). Além disso, temse demonstrado um aumento na procura de produtos vegetarianos. São considerados vegetarianos aqueles que excluem qualquer alimento de origem animal do padrão alimentar, incluindo nesta restrição todos os tipos de carnes, aves, peixes e seus derivados, como ovos e leite (HARGREAVES et al., 2020). Logo, demanda-se das indústrias o desenvolvimento de produtos com características físico-químicas e nutricionais capazes de propiciar benefícios à saúde e que ainda sejam agradáveis ao paladar (BARBOSA; CAZAL, 2018).

A oliveira (Olea europaea L.) é uma frutífera da família botânica Oleaceae. Além do azeite e da azeitona de mesa, a oliveira é composta de folhas que representam $10 \%$ do peso das azeitonas coletadas para a extração do óleo (HERRERO et al., 2011). Essas folhas são consideradas subprodutos da indústria e ainda são pouco explorados. As folhas da oliveira, mistura entre as folhas e galhos que costumam ser descartados, possuem um

\section{Revista Verde}

ISSN 1981-8203

Pombal, Paraíba, Brasil v. 16, n.2, abr.-jun, p.159-163, 2021

doi: $10.18378 /$ rvads.v16i2.8385

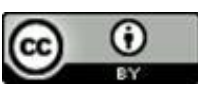


potencial antioxidante pela presença de polifenóis (ACAR-TEK; AĞAGÜNDÜZ, 2020), principalmente a oleuropeína e o hidroxitirosol (HALOUI et al., 2011). Recentemente, estudos retrataram que as folhas de oliveira contêm maior quantidade de polifenóis do que o azeite de oliva (VOGEL et al., 2015).

As folhas de oliveira, resultantes da colheita mecanizada, quando descartadas de maneira inadequada podem causar grande impacto ambiental, por isso torna-se essencial o desenvolvimento de métodos de reaproveitamento das folhas. Uma forma de remanejar as folhas de oliveira é o desenvolvimento de coprodutos a partir dos mesmos, reduzindo os danos ao meio ambiente causado pelo descarte incorreto, além de agregar valor nutricional aos apreciadores de produtos com redução de impacto ambiental (SANTOS et al., 2012; BEN SALEM et al., 2015). Um estudo recente publicado por Faccioli et al. (2021) produziu com subproduto das folhas de oliveira o desenvolvimento de um biscoito salgado, do tipo cracker, demonstrando um bom índice de aceitabilidade e intenção de compra entre os painelistas treinados.

Nesse contexto, o objetivo deste trabalho foi desenvolver uma versão vegetariana de pão de queijo, elaborado com ingredientes exclusivamente in natura e minimamente processados de origem vegetal adicionado de farinha das folhas de oliveira (FFO) reaproveitadas.

\section{MATERIAL E MÉTODOS}

\section{Matérias-primas}

As folhas de oliveira utilizadas para este estudo foram fornecidas pela Fazenda Experimental, localizada no município de Barra do Ribeiro-RS, Brasil (Latitude: -30.2915, Longitude: $51.302630^{\circ} 17^{\prime} 29^{\prime \prime} \mathrm{Sul}, 51^{\circ} 18^{\prime} 9^{\prime \prime}$ Oeste). Os demais ingredientes (batata inglesa, polvilho doce, polvilho azedo, água, azeite de oliva extravirgem, sal refinado e pimenta da jamaica) utilizados para a elaboração do pão de queijo foram adquiridos em um estabelecimento comercial localizado na cidade de Porto Alegre-RS, Brasil.

\section{Obtenção da farinha das folhas de oliveira (FFO)}

Para a obtenção da FFO, as folhas colhidas foram selecionadas, separadas do caule e pesadas. Para a lavagem, as folhas foram imersas em água deionizada durante 15 minutos, por duas vezes. Após esse processo, as folhas foram submersas em uma solução com hipoclorito de sódio a $1 \%$ para a higienização e enxaguadas em água corrente durante 30 minutos.
Depois foram lavadas novamente com água deionizada durante 15 minutos, por duas vezes. As folhas lavadas foram secas em superfície de aço inoxidável a uma temperatura média de $37{ }^{\circ} \mathrm{C}$ por 48 horas. As folhas secas foram submetidas à moagem utilizando o multiprocessador Thermomix Vorwerk (® (Wuppertal, Renânia do Norte-Vestfália, Alemanha). Este foi utilizado na temperatura de $37^{\circ} \mathrm{C}$ até a obtenção da farinha. Após o processo de moagem, o produto foi peneirado em peneira de aço inoxidável manual $(250 \mu \mathrm{m}) \mathrm{com}$ a peneira granulométrica Inox 8X2 abertura 0,5 mm malha 35 (São Paulo, São Paulo, Brasil). A farinha foi armazenada em recipiente de vidro com tampa devidamente armazenada até o momento da utilização.

\section{Elaboração do pão de queijo adicionado de farinha das folhas de oliveira}

A formulação deste trabalho é a releitura do pão de queijo tradicional, feito à base de batata inglesa e sem adição de queijo. O pão de queijo foi escolhido como preparação culinária deste estudo, na intenção de apresentar uma opção de lanche com uma composição diferente do habitual, porém com atributos sensoriais comuns ao paladar dos brasileiros.

A elaboração dos pães foi realizada no Laboratório de Cozinha Experimental, do Curso de Nutrição da Universidade Federal de Ciências da Saúde de Porto Alegre (UFCSPA). Testes preliminares foram realizados como um screening para definir a proporção mais adequada dos ingredientes capazes de proporcionar sabor e textura adequados. Após os testes preliminares, obteve-se uma formulação padrão, a partir da qual foram estabelecidas as novas formulações com composição alterada. Foi considerado versão vegetariana a formulação produzida a partir da versão de pão de queijo sem adição do queijo e adição de batata inglesa branca. Sendo a partir desta, considerada a adição da FFO.

A partir da Formulação A (controle), foram elaboradas quatro formulações (B, C, D e E) com adição de FFO nas concentrações de $0,95 \%, 1,59 \%, 2,23 \%$ e $2,85 \%(\mathrm{~m} / \mathrm{m})$, respectivamente, as quais são listadas na Tabela 1. Para a receita manter a textura de pão de queijo, com exceção da FFO, a quantidade dos outros ingredientes não foram alteradas. Assim, a inclusão da FFO foi calculada sob o percentual de ingredientes totais e realizado porcentagem sobre esse valor, dessa forma a FFO foi adicionada a Formulação A, sem substituição de nenhum dos ingredientes, por esta razão o percentual de ingredientes das Formulações B, C, D e E são superiores a 100\%. As formulações utilizadas podem ser observadas na tabela 1 .

Tabela 1. Formulações da Versão Vegetariana de Pães de Queijo adicionado de farinha das folhas de oliveira

\begin{tabular}{lccccc}
\hline & \multicolumn{5}{c}{ Formulações } \\
\cline { 2 - 6 } Ingredientes & $\mathrm{A}$ & $\mathrm{B}$ & $\mathrm{C}$ & $\mathrm{D}$ & $\mathrm{E}$ \\
\hline Batata Inglesa Cozida (\%) & 45,85 & 45,85 & 45,85 & 45,85 & 45,85 \\
Polvilho Azedo (\%) & 21,48 & 21,48 & 21,48 & 21,48 & 21,48 \\
Polvilho Doce (\%) & 10,36 & 10,36 & 10,36 & 10,36 & 10,36 \\
Água (\%) & 18,45 & 18,45 & 18,45 & 18,45 & 18,45 \\
Azeite de Oliva Extra Virgem (\%) & 2,39 & 2,39 & 2,39 & 2,39 & 2,39 \\
Sal Refinado (\%) & 1,06 & 1,06 & 1,06 & 1,06 & 1,06 \\
Pimenta da Jamaica (\%) & 0,41 & 0,41 & 0,41 & 0,41 & 0,41 \\
Farinha de Folha de Oliveira (\%) & 0 & 0,95 & 1,59 & 2,23 & 2,85 \\
\hline TOTAL (\%) & 100 & 100,95 & 101,59 & 102,23 & 102,85 \\
\hline
\end{tabular}




\section{Processamento dos pães de queijo}

Para a elaboração dos pães de queijo, primeiramente, todos os ingredientes foram pesados em balança eletrônica digital Even ${ }^{\circledR}$ BL3200AS-BI. Após pesagem, as batatas foram cozidas em água previamente aquecida em fogão industrial a gás, por aproximadamente 20 minutos, até ficarem em consistência possível de amassar manualmente. Em seguida, as batatas foram descascadas com descascador de legumes DB6 Metvisa e amassadas manualmente. Logo após, foram incorporados os demais ingredientes (polvilhos, sal, pimenta, água e óleo), sendo manuseados até a obtenção de uma massa homogênea. A massa foi dividida em pães de queijo com aproximadamente $25,8 \mathrm{~g}$ e assados durante 25 min em forno turbo FTT $80 \mathrm{E}$ à $140{ }^{\circ} \mathrm{C}$.

\section{Análise sensorial}

Para analisar de forma mais completa os aspectos do produto, a metodologia utilizada foi a técnica de Análise Descritiva Quantitativa (ADQ). Esta técnica permite avaliar o perfil sensorial quanto aos atributos, sendo estes, variáveis conforme o produto analisado. Após aplicados, os testes de ADQ resultam em uma descrição completa do produto, sendo seus resultados a base para mapear as semelhanças e disparidades entre as amostras, além de permitir realizar correlações entre ingredientes ou variáveis do processo de produção com os atributos sensoriais (SILVA et al, 2014). É uma metodologia de avaliação da intensidade de atributos sensoriais previamente definidos, e não de aceitabilidade. Os atributos devem ter relação com a amostra controle e com os processos ou ingredientes em avaliação, assim em uma escala de $9 \mathrm{~cm}$ pode-se verificar a alta ou baixa intensidade em que os atributos selecionados foram percebidos pelos avaliadores, sendo $0 \mathrm{~cm}$ baixa até $9 \mathrm{~cm}$ muito intensa, caracterizando assim o perfil sensorial das amostras.

Os atributos foram definidos a partir de uma triagem com os painelistas treinados. Os atributos definidos como relevantes no produto foram: aroma polvilho, aroma herbáceo, aroma queijo, sabor amargo, sabor herbáceo, sabor salgado, sabor picante, sabor queijo, textura macia, textura dureza, textura elasticidade, textura granulosidade, cor esverdeado, cor marrom e cor bege.

A avaliação sensorial dos produtos foi realizada por 8 painelistas treinados, sendo 5 mulheres e 3 homens, com idade entre 20 e 57 anos. A análise foi realizada em cabines individuais sob luz branca e a temperatura ambiente de $22{ }^{\circ} \mathrm{C}$, com as amostras dispostas em pires brancos de porcelana. As amostras, em porções padronizadas (aproximadamente $25 \mathrm{~g}$ ), foram codificadas com números aleatórios de 3 dígitos e apresentadas aos avaliadores de forma randomizada. Acompanhado das amostras, foram entregues água mineral a temperatura ambiente de $22{ }^{\circ} \mathrm{C}$ para limpeza do palato e a ficha do teste contendo escalas de $10 \mathrm{~cm}$, utilizadas pelos painelistas para a avaliação do produto quanto a intensidade dos atributos previamente definidos.

O presente trabalho foi aprovado pelo Comitê de Ética em Pesquisa (CEP) da UFCSPA sob o registro número $n$. 12165219.5.0000.5345, onde todos os participantes assinaram o Termo de Consentimento Livre e Esclarecido.

\section{Análise estatística}

O delineamento experimental foi o de blocos casualizados. Os dados obtidos na análise sensorial das formulações, foram analisados por meio de Análise de Variância (ANOVA) de um fator, utilizando média e desvio padrão. Para a comparação das médias entre as amostras, foi utilizado o teste de Tukey, ao nível de 5\% de significância. Para a análise dos dados foi utilizado o software SPSS Statistics versão 22.

\section{RESULTADOS E DISCUSSÃO}

Os atributos selecionados foram aroma polvilho, aroma herbáceo, aroma queijo, sabor amargo, sabor herbáceo, sabor salgado, sabor picante, sabor queijo, textura macia, textura dureza, textura elasticidade, textura granulosidade, cor esverdeado, cor marrom e cor bege. A partir deste foi efetuada a caracterização do perfil sensorial das amostras, cujas médias de cada atributo avaliado encontram-se na Tabela 2.

A partir da diferença estatística das notas médias entre as Formulações A e E (Tabela 2), verificou-se quatro atributos sensoriais com variações perceptíveis pelos painelistas: aroma herbáceo, sabor amargo, sabor herbáceo e sabor queijo. Entre estas, a adição de FFO demonstrou maior influência no atributo sabor amargo, com médias variando entre 1,06 (Formulação A) e 6,23 (Formulação E). Este atributo torna-se mais evidente conforme o aumento da adição de FFO, o que era esperado visto que a oleuropeína, presente nas folhas da oliveira, é um glicosídeo amargo (COPPA et al., 2017).

Assim como sabor amargo, os atributos sabor e aroma herbáceos também apresentaram maiores médias à medida que aumentam as quantidades de FFO, estes obtiveram variação entre 0,82 (Formulação A) a 5,21 (Formulação E) e, entre 0,88 (Formulação A) e 4,47 (Formulação E), respectivamente. A diferença estatística encontrada nos atributos aroma e sabor herbáceo também era esperada, tendo em vista que estes são atributos relacionados apenas ao ingrediente FFO, incomum a formulação de pão de queijo controle. Estes atributos obtiveram diferença significativa entre si, mantendo semelhança entre as formulações A, B, C e D, o que sugere que até $2,23 \%$ de adição de FFO, os atributos de aroma herbáceo e sabor herbáceo não são perceptíveis quando comparados a formulação controle, sendo detectada esta diferença quando a concentração de FFO adicionada é superior a 2,85\%.

Os dados demonstram que conforme a concentração de FFO é aumentada, os atributos relacionados a este ingrediente sofrem modificação, dessa forma pode-se observar que as maiores médias nos atributos sensoriais citados (aroma herbáceo, sabor amargo e sabor herbáceo) das Formulações B, C, D e E foram proporcionais ao aumento na concentração de FFO. Assim como os resultados encontrados por Ataei e Hojjatoleslamy (2017), ao avaliar as características sensoriais de um pão de ló feito com FFO, observaram que os atributos relacionados ao sabor e aroma do pão de ló, entre às suas formulações, também demonstraram diferença estatística, do mesmo modo que entre as formulações de pão de queijo, de $\mathrm{A}$ a $\mathrm{E}$, descritas nesse estudo. Em suas amostras, também não substituíram parte dos ingredientes por FFO, apenas adicionaram. 
Tabela 2. Médias de intensidade dos atributos sensoriais das amostras de pão de queijo na versão vegetariana adicionado de Farinha de Folha de Oliveira

\begin{tabular}{|c|c|c|c|c|c|c|}
\hline \multirow[t]{2}{*}{ Atributos } & \multicolumn{5}{|c|}{ Formulações } & \multirow[t]{2}{*}{$p$-valor } \\
\hline & A & B & $\mathrm{C}$ & $\mathrm{D}$ & $\mathrm{E}$ & \\
\hline Aroma Polvilho & $4,08 \pm 2,59$ & $3,55 \pm 2,62$ & $3,53 \pm 2,69$ & $2,73 \pm 2,17$ & $2,76 \pm 2,7$ & 0,803 \\
\hline Aroma Herbáceo & $0,88 \pm 1,07^{\mathrm{a}}$ & $1,87 \pm 1,38^{\mathrm{a}}$ & $2,7 \pm 2,17^{\mathrm{a}}$ & $3,48 \pm 1,73^{\mathrm{a}}$ & $4,47 \pm 3,15^{\mathrm{b}}$ & 0,013 \\
\hline Aroma Queijo & $2,55 \pm 1,20$ & $2,03 \pm 1,53$ & $1,96 \pm 1,86$ & $2,33 \pm 1,39$ & $1,26 \pm 0,95$ & 0,450 \\
\hline Sabor Amargo & $1,06 \pm 1,05^{\mathrm{a}}$ & $3,47 \pm 3,13^{\mathrm{a}}$ & $4,80 \pm 2,66^{\mathrm{a}}$ & $5,22 \pm 2,86^{\mathrm{a}}$ & $6,23 \pm 3,51^{b}$ & 0,008 \\
\hline Sabor Herbáceo & $0,82 \pm 0,94^{\mathrm{a}}$ & $4,06 \pm 2,75^{\mathrm{a}}$ & $4,07 \pm 3,36^{\mathrm{a}}$ & $4,80 \pm 2,51^{\mathrm{a}}$ & $5,21 \pm 3,17^{b}$ & 0,021 \\
\hline Sabor Salgado & $3,41 \pm 0,89$ & $3,53 \pm 1,27$ & $3,08 \pm 1,09$ & $3,0 \pm 1,28$ & $3,05 \pm 1,39$ & 0,862 \\
\hline Sabor Picante & $1,11 \pm 1,68$ & $1,55 \pm 1,85$ & $0,93 \pm 1,0$ & $1,67 \pm 1,62$ & $2,21 \pm 2,83$ & 0,693 \\
\hline Sabor Queijo & $3,0 \pm 1,08$ & $2,53 \pm 1,34$ & $1,45 \pm 1,16$ & $1,97 \pm 1,28$ & $1,33 \pm 0,98$ & 0,036 \\
\hline Textura Macia & $4,01 \pm 1,56$ & $4,11 \pm 1,21$ & $5,95 \pm 1,91$ & $4,73 \pm 2,48$ & $5,32 \pm 2,26$ & 0,246 \\
\hline Textura Dureza & $3,13 \pm 1,25$ & $2,81 \pm 1,60$ & $1,67 \pm 1,56$ & $1,76 \pm 1,52$ & $2,53 \pm 2,39$ & 0,355 \\
\hline Textura Elasticidade & $3,75 \pm 1,89$ & $4,22 \pm 1,68$ & $5,26 \pm 2,17$ & $4,12 \pm 2,27$ & $4,47 \pm 2,13$ & 0,658 \\
\hline Textura Granulosidade & $0,86 \pm 0,93$ & $1,42 \pm 1,51$ & $1,22 \pm 1,52$ & $1,71 \pm 2,15$ & $1,36 \pm 1,46$ & 0,867 \\
\hline Cor Esverdeado & $0,62 \pm 0,97$ & $1,32 \pm 2,27$ & $1,58 \pm 1,32$ & $2,32 \pm 1,20$ & $2,50 \pm 2,33$ & 0,202 \\
\hline Cor Marrom & $2,96 \pm 1,96$ & $4,30 \pm 2,03$ & $4,47 \pm 1,40$ & $4,87 \pm 2,36$ & $5,78 \pm 2,49$ & 0,127 \\
\hline Cor Bege & $5,69 \pm 1,05$ & $5,02 \pm 1,01$ & $5,50 \pm 2,08$ & $4,17 \pm 2,57$ & $2,66 \pm 1,96$ & 0,015 \\
\hline
\end{tabular}

Formulação A = 0\% de FFO; Formulação B = 0,95\% de FFO; Formulação C = 1,59\% de FFO; Formulação D = 2,23\% de FFO; Formulação E = 2,85\% de Farinha de Folha de Oliveira. Médias com letras diferentes na mesma linha apresentam diferença significativa entre si $(\mathrm{p} \leq 0,05)$, conforme resultado do teste de Tukey.

A amostra elaborada sem FFO (Formulação A) apresentou médias superiores nos atributos aroma de polvilho e cor bege, sendo estas 4,08 e 5,69, respectivamente. Estas médias foram diminuindo gradativamente até a maior concentração de FFO (Formulação E), atingindo a média de 2,76 para aroma de polvilho e 2,66 para cor bege.

Quanto aos atributos relacionados à textura, as médias mais altas foram para textura macia e elasticidade, porém, não foram encontradas distinções entre as formulações. A textura não obteve influência bem como os atributos aroma e sabor, isso se dá em virtude da FFO ser um ingrediente em adição e não em substituição dos polvilhos totais. Desse modo, é possível inferir que a textura das formulações A, B, C, D e E se mantiveram sem alterações pela manutenção da mesma quantidade dos demais ingredientes (polvilho azedo, polvilho doce, batata, água, azeite, sal e pimenta).

Ao realizar análise sensorial ou mesmo a degustação de alimentos, às percepções dos avaliadores iniciam-se nos olhos antes que na língua. Ao definir os atributos deste alimento e suas intensidades, muitas informações são construídas: o sabor que se sente quando o alimento atinge às papilas gustativas, a construção desse sabor, às referências afetivas e culturas desse paladar (ALTOÉ et al., 2019).

O sabor queijo apresentou diferença estatística significativa $(p \leq 0,05)$ entre as amostras, embora não houvesse queijo entre os ingredientes em nenhuma das formulações, sendo a formulação A a qual obteve maiores valores. A alimentação tem a capacidade de imprimir marcas na memória (BOGADO; FREITAS, 2016), nesse sentido a intenção de atribuir o sabor queijo às formulações de pão de queijo foi exatamente avaliar em que intensidade esta referência, relacionada aos atributos do pão de queijo tradicional, era percebida pelos avaliadores.

$\mathrm{O}$ valor nutricional do produto final foi estimado via software para nutricionistas, sugere-se que para trabalhos futuros a análise centesimal de produtos seja realizada.

$\mathrm{O}$ valor nutricional foi estimado pelo programa Dietbox, onde cada unidade de pão de queijo (25,8 g) contém $7,9 \mathrm{~g}$ de carboidratos, $0,16 \mathrm{~g}$ de proteínas, $0,69 \mathrm{~g}$ de lipídios totais, sendo $0,1 \mathrm{~g}$ saturados, $0,52 \mathrm{~g}$ monoinsaturados e $0,06 \mathrm{~g}$ poliinsaturados. As quantidades de polifenóis e oleuropeína, por unidade, variaram de $0,02 \mathrm{~g}$ e $0,44 \mathrm{~g}$ (Formulação B) a $0,06 \mathrm{~g}$ e 1,34 g (Formulação E), respectivamente. 


\section{CONCLUSÃO}

As características sensoriais da versão vegetariana de pão de queijo com farinha de oliveira evidenciam que o aumento da quantidade de FFO destaca o sabor amargo, sabor e aroma herbáceos, enquanto os atributos das texturas e o sabor salgado não sofrem mudanças, sendo um potencial a ser utilizado na culinária.

\section{REFERÊNCIAS}

ACAR-TEK, N.; AĞAGÜNDÜZ D. Olive leaf (Olea europaea L. folium): Potential effects on glycemia and lipidemia. Annals of Nutrition and Metabolism, v.76, n. 1, p.10-15, 2020. $\underline{10.1159 / 000505508}$

ATAEI, F.; HOJJATOLESLAMY, M. Physicochemical and sensory characteristics of sponge cake made with olive leaf. Journal of Food Measurement and Characterization, v.11, p.2259-64, 2017. 10.1007/s11694-017-9610-6

ALTOÉ, I.; MENOTTI, G.; AZEVEDO, E. D.; Comida e afeto: As releituras dos pratos-totem na culinária vegana. Revista Brasileira de Sociologia da Emoção, v.18, n. 52, p. 129-138, 2019.

BARBOSA, H. C.; CAZAL, M. M.; Evaluation of the influence of the sensory characteristics and nutritional knowledge in the acceptance of mate tea. Brazilian Journal of Food Technology, v.21, e2017075, 2018. 10.1590/1981-6723.7517

BEN SALEM, M.; AFFES, H. KSOUDA, K.; SAHNOUN, Z.; ZEGHAL, K. M.; HAMMAMI, S. Pharmacological Activities of Olea europaea Leaves. Journal of Food Processing and Preservation, v.39, n. 6, p.3128-36, 2015. 10.1111/jfpp.12341

BOGADO, A. M.; FREITAS, D. A reconstrução de memórias da alimentação na formação inicial de professores de ciências: Um reencontro com saberes, sabores, aromas e afetos. Revista Educação Temática Digital, v.18, n.3, p.670-689, 2016. 10.20396/etd.v18i3.8645410
COPPA, C. F. S. C.; ROSIM, R. E.; OLIVEIRA, C. A. F.; RODRIGUES, C. E. C.; GONÇALVES, C. B. Extraction of oleuropein from olive leaves using a hydroalcoholic solvent. Brazilian Journal of Food Technology, v.20, e.2016169, p.1-9, 2017. 10.1590/1981-6723.16916

FACCIOLI, L. S.; KLEIN, M. P.; BORGES, G. R.; DALANHOL, C. S.; MACHADO, I. S. K.; GARAVAGLIA, J.; DAL BOSCO, S. M. Development of crackers with the addition of olive leaf flour (Olea europaea L.): Chemical and sensory characterization. LWT - Food Science and Technology, v.141, p.1108-48, 2021. 10.1016/j.lwt.2021.110848

HARGREAVES, S. M.; NAKANO, E. Y.; ZANDONADI, R. P.; Brazilian vegetarian population - Influence of type of diet, motivation and sociodemographic variables on quality of life measured by specific tool (VEGQOL). Nutrientes, v.12, n, 5, p.1406-1428, 2020. 10.3390/nu12051406

HERRERO, M. T. N.; TEMIRZODA, A.; SEGURACARRETERO, R.; QUIRANTES, M.; PLAZA, E. New possibilities for the valorization of olive oil by-products. Journal of Chromatography A, v.1218, n.42, p.7511-7520, 2011. $\underline{10.1016 / j . c h r o m a .2011 .04 .053}$

SANTOS, L.; KOTOVICZ, V.; BARANA, A. C.; ALMEIDA, M. M.; Utilização de resíduos agroindustriais para produção de amiloglucosidase por Aspergillus awamori. Revista Brasileira de Tecnologia Agroindustrial, v. 6, n.1, p.655-664, 2012. $\underline{10.3895 / \text { S1981-36862012000100004 }}$

SILVA, P. P. M.; CASEMIRO, R. C.; ZILLO, R. R.; CAMARGO, A. C.; PROSPERO, E. T. P.; SPOTO, M. H. F. Sensory descriptive quantitative analysis of unpasteurized and pasteurized jucara pulp (Euterpe edulis) during long-term storage. Food Science \& Nutrition, v.2, n.4, p. 321- 331, 2014. $10.1002 / \mathrm{fsn} 3.105$

VOGEL, P.; MACHADO, I. K.; GARAVAGLIA, J.; ZANI, V. T.; SOUZA, D.; DAL BOSCO, S. M.; Polyphenols benefits of olive leaf (Olea europaea L.) to human health. Nutrición Hospitalaria, v.31, n.3, p.1427-1433, 2015. 10.3305/nh.2015.31.3.8400 